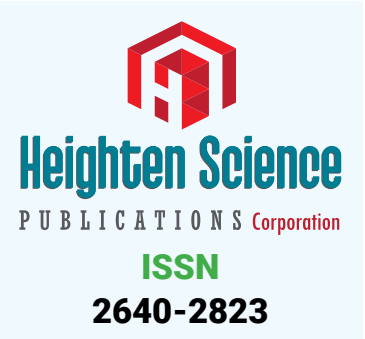

*Address for Correspondence: Dr. Peng Luo, Department of Respiratory Medicine, Zhujiang Hospital, Southern Medical University, 253\# Industry road, Guangzhou, Guangdong, China, Tel: +86 13246269394, Fax: +86 02061643888; Email: smuluopeng@163.com

Submitted: 27 February 2017

Approved: 28 March 2017

Published: 30 March 2017

Copyright: @ 2017 Zhang X et al. This is an open access article distributed under the Creative Commons Attribution License, which permits unrestricted use, distribution, and reproduction in any medium, provided the original work is properly cited

Keywords: Neutrophil to Lymphocyte Ratio; NLR; Prognostic Predictor; Systematic Inflammation; Non-small Cell Lung Cancer; NSCLC

Check for updates
Editorial

\section{Neutrophil to Lymphocyte Ratio (NLR) in Peripheral Blood: A Novel and Simple Prognostic Predictor of Non-small Cell Lung Cancer (NSCLC)}

\author{
Xiaoli Zhang, Ziyuan Zou, Liyu Fan, Xinjie Xu, Yu Siyuan and \\ Peng Luo*
}

Department of Respiratory Medicine, Zhujiang Hospital, Southern Medical University, Guangzhou 510282, P. R. China

\section{EDITORIAL}

Lung cancer is the leading cause of cancer-related deaths worldwide, and almost accounts for $20 \%$ of these deaths, however, the cure rate is less than $10 \%$ [1]. Non-small cell lung cancer (NSCLC) accounts for approximately $85 \%$ of all cases of lung cancer [1], but fewer than $15 \%$ of individuals diagnosed with NSCLC can survive for more than 5 years, which poses a great threat to the patient's life and health [2]. Recently, the incidence of lung cancer keeps dynamically growing, but more than $75 \%$ of patients at diagnosis has appeared local development or metastasis, missing the best period of surgery. Moreover, despite surgical treatment is the optimal choice for early-stage NSCLC patients, 30\%-40\% of patients with NSCLC develop tumor recurrence in a short time. Therefore, improving the prognosis of patients with lung cancer and predicting the long-term survival of patients is of particular importance [3]. At present, tumor and node metastasis (TNM) staging system, clinicopathological characteristics, visceral pleural invasion and marginal status are used to predict the disease progression and overall survival of NSCLC patients. There is no index which is stable, effective, reliable and less harmful to assess prognosis, predict recurrence risk and overall survival.

The genesis, progress and metastasis of NSCLC are not only related to the intrinsic characteristics of cancer cells but also to the cancer microenvironment. Systemic inflammation is a promoter of tumor genesis. Cumulative evidence has revealed that tumor-promoting inflammation contributes to cancer growth and progression by creating a tumor-promoting microenvironment, stimulates the proliferation of bronchial stem cells, and triggers the carcinogenesis of lung epithelial cell. Additionally, systematic inflammatory can not only cause tumor growth and promote tumor metastasis by activating type $2 \mathrm{~T}$-assisted response, chemokines, vascular regeneration and others [4], but also responsible for reducing the effect of anti-cancer drugs and cancer-related symptoms including anorexia, cancerous pain, debilitation, cachexia [2]. In total, systematic inflammation has been shown to play an important impact on the pathogenesis and progression of NSCLC and is of great value to the aggravation and prognosis of NSCLC.

There are several promising and reliable Predictors of systemic inflammation, such as C-reactive protein, albumin, fibrinogen, erythrocyte sedimentation rate, while recent 
studies showed that the neutrophils to lymphocytes ratio in peripheral blood is the most representative indicator of systematic inflammation, which might be caused by following reasons. Firstly, being dehydrated, in vitro preparation of blood samples and other factors will affect the levels of C-reactive protein, neutrophils or lymphocytes. Moreover, catecholamines will lead to the release of neutropenia and lymphopenia, but those factors have little impact on the levels NLR. Ultimately, NLR also has the advantages of simplicity, easy calculation and convenience. Thus, NLR is becoming a novel and a popular prognostic indicator of NSCLC, besides, it has great application prospect in the diagnosis, prognosis and clinical treatment of NSCLC [5].

A recent meta-analysis, which pooled 3,656 NSCLC patients from 14 studies and high NLR (especially with a cutoff of 5) seems to be associated with a worse prognosis in patients with NSCLC as well as a worse response to treatments [6]. The five-year survival rate in patients with a lower NLR level is about $62 \%$, while the overall survival of patients with high NLR levels is between 1.39-2.09 [5]. This can be explained by the followings: higher NLR levels mean the increased neutrophil levels, which indicates the body is in a more serious non-specific inflammatory state, the diminished lymphocyte levels indicate the body is not sensitive to immune response and in poor immune protection. Neutrophils played a double-edged role in tumor development. On one hand, CD11b +/Ly6G+ tumor-associated neutrophils are toxic to tumor cells. On the other hand, neutrophils can promote tumor metastasis through Mac-1-mediated pathways, secrete vascular endothelial growth factor, and inhibit the dissolution activity of lymphatic cells, natural killer cells, contributing to tumor proliferation [2]. Most of the neutrophils play a role in promoting tumor growth and immunosuppression [2], while the loss of neutrophils may inhibit tumor growth. As the host's immune response to the tumor is lymphocyte-dependent, lymphocytes can not only induce cytotoxin-mediated apoptosis but also produce a certain cytokine to inhibit tumor cell proliferation and migration. The relative lower lymphocytes level will undoubtedly reduce the immune response to the body, and increase the risk of tumor cells invading the body [7]. NLR is more accurate to reflect the imbalance of tumor and antitumor activity in the inflammatory response than the single use of neutrophils or lymphocytes.

Although NLR has been demonstrated as a clinical indicator of NSCLC prognosis in several preliminary studies, more multicenter large-scale prospective studies need to be performed to determine the specific cut-off value and diagnostic capabilities of NLR. There are several potential benefits for NSCLC patients, which might be the mainstreams of NLR and NSCLC: (1). Combining NLR with other independent predictors, a comprehensive efficacy or prognosis assessment scale could be formulated; (2). Long-term monitoring of systemic inflammation could be continuously measured in NSCLC patients and intervene timely; (3) According to the levels of NLR to evaluate the systematic inflammatory status in NSCLC patients, that evaluation will contribute to preoperative or postoperative treatment to achieve a better therapeutic effect.

\section{REFERENCES}

1. Dirican N, Dirican A, Anar C, Atalay S, Ozturk O, et al. A New Inflammatory Prognostic Index, Based on C-reactive protein, the Neutrophil to Lymphocyte Ratio and Serum Albumin is Useful for Predicting Prognosis in Non-Small Cell Lung Cancer Cases. Asian Pac J Cancer Prev. 2016; 17: 5101-5106. Ref.: https://goo.gl/k3vLfQ

2. Wang J, Kalhor N, Hu J, Wang B, Chu H, et al. Pretreatment Neutrophil to Lymphocyte Ratio Is Associated with Poor Survival in Patients with Stage I-III Non-Small Cell Lung Cancer. PloS one. 2016; 11. Ref.: https://goo.gl/GjnBc1

3. Jin F, Han A, Shi F, Kong L, Yu J, et al. The postoperative neutrophil-to-lymphocyte ratio and changes in this ratio predict survival after the complete resection of stage I non-small cell lung cancer OncoTargets ther. 2016; 9: 6529-6537. Ref.: https://goo.gl/tAJlnN 
4. Tomita M, Shimizu T, Ayabe T, Nakamura K, Onitsuka T, et al. Elevated preoperative inflammatory markers based on neutrophil-to-lymphocyte ratio and C-reactive protein predict poor survival in resected non-small cell lung cancer. Anticancer res. 2012; 32: 3535-3538. Ref.: https://goo.gl/ZM93k9

5. Gu XB, Tian T, Tian XJ, Zhang XJ. Prognostic significance of neutrophil-to-lymphocyte ratio in nonsmall cell lung cancer: a meta-analysis. Sci Rep. 2015; 5: 12493. Ref.: https://goo.gl/jOfxEL

6. Peng $B$, Wang $Y H, L i u Y M, M a ~ L X$. Prognostic significance of the neutrophil to lymphocyte ratio in patients with non-small cell lung cancer: a systemic review and meta-analysis. Int $\mathrm{J}$ Clin Exp Med. 2015; 8: 3098-3106. Ref.: https://goo.gl/XJ7Prw

7. Eryilmaz M K, Mutlu H, Salim D K, Musri FY, Tural D, et al. The neutrophil to lymphocyte ratio has a high negative predictive value for pathologic complete response in locally advanced breast cancer patients receiving neoadjuvant chemotherapy. Asian Pac J Cancer Prev. 2014; 15: 7737-7740. Ref.: https://goo.gl/vgeXCu 